\title{
Novel targeted therapy for acute myeloid leukemia with a dual FLT3 and JAK2 inhibitor
}

\author{
Yin-jun LOU*
}

Acta Pharmacologica Sinica (2012) 33: 212-213; doi: 10.1038/aps.2011.206; published online 9 Jan 2012

A cute myeloid leukemia (AML) is a highly malignant hematopoietic tumor. The use of all-trans retinoic acid (ATRA) and arsenic trioxide, which began from China, has resulted in revolution of the acute promyelocytic leukemia (APL) that appears curable in more than $70 \%$ of patients ${ }^{[1]}$. However, the treatment regimen for nonAPL AML particularly in older patients has progressed little in the past two decades. Intensive efforts have been made toward the development of novel target agents, which are based on newfound pathophysiological events crucial for cancers. FMS-related tyrosine kinase 3 (FLT3), which is mutated in about $30 \%$ patients of $\mathrm{AML}$, is the most ideal target for molecular therapy. Over the past decade, a number of FLT3 inhibitors have been studied in clinical trials. However, the results were often transient because of secondary resistance developed $^{[2]}$. In addition, genetic lesions and aberrations involving Janus-associated kinase 2 (JAK2) have been found to be associated with a wide spectrum of hematological malignancies ${ }^{[3]}$. Recently, $S$ Hart et al reported that pacritinib (SB1518), a dual JAK2/FLT3 inhibitor, emerged as an ideal new therapeutic

Department of Hematology, Institute of Hematology, the First Affiliated Hospital of Zhejiang University, School of Medicine, Hangzhou 310003, China Correspondence: Dr Yin-jun LOU

(louyinjun@yahoo.com.cn) agent for acute myelogenous leukemia in a preclinical study ${ }^{[4]}$.

Pacritinib is a potent and selective inhibitor of FLT3 and JAK2 with $\mathrm{IC}_{50}$ of 23 and $22 \mathrm{nmol} / \mathrm{L}$, respectively ${ }^{[5]}$. In the study, the authors demonstrated pacritinib led to a dose-dependent decrease of FLT3 auto-phosphorylation and downstream effectors of STAT5, ERK1/2, AKT phosphorylation in FLT3internal-tandem duplication (ITD) cell lines (MV4-11, MOLM-13) and in FLT3wt-bearing cell line (RS4;11). The agent inhibited the proliferation of MV4-11, MOLM-13 and RS4; 11 cells with $\mathrm{IC}_{50}$ of 47,67, and $930 \mathrm{nmol} / \mathrm{L}$, respectively. Furthermore, the JAK2V617F-harboring cell line, SET-2, was also very sensitive to pacritinib $\left(\mathrm{IC}_{50}=220 \mathrm{nmol} / \mathrm{L}\right)$. Flow cytometry analysis showed that the agent could induce $G_{1}$ arrest and caspase-dependent apoptotsis. Pacritinib inhibited the proliferation of 14 primary AML samples with the $\mathrm{IC}_{50}$ ranging from $190 \mathrm{nmol} / \mathrm{L}$ to $1300 \mathrm{nmol} / \mathrm{L}$, with concomitant inhibition of phosphorylation of FLT3, STAT3, and STAT5. The two samples harboring the FLT3-ITD mutation were among the most sensitive. Moreover, pacritinib was also highly active in in vivo models of FLT3ITD driving cell lines. In MV4-11 tumorbearing mice, pacritinib (once daily for 21 consecutive days) induced dosedependent inhibition of tumor growth. Complete regression was observed in $3 / 10$ and $8 / 8$ mice in the groups receiving 50 and $100 \mathrm{mg} \cdot \mathrm{kg}^{-1} \cdot \mathrm{day}^{-1}$, respectively. In the MOLM-13 model, pacritinib treatment $(150 \mathrm{mg} / \mathrm{kg}$ bid for 7 consecutive days) resulted in tumor growth inhibition of $83 \%$. Finally, higher activity of JAK/STAT signaling was confirmed in FLT3-linifanib/ABT869 resistant cells (MV4-11-R). Pacritinib was highly effective in the resistant cell lines. A combination of FLT3 inhibitor linifanib with JAK family inhibitor ruxolitinib showed the synergistic effect on MV4-11 cells.

Interestingly, pacritinib entered the clinic in 2008 has completed Phase 2 trials for myelofibrosis. It has shown promising clinical activity and a favorable safety profile. The agent has received orphan drug designation from the US and the EU regulatory authorities. In summary, the preliminary results of the dual inhibitor of FLT3 and JAK2 were promising. Hope remains that pacritinib will be become an effective therapeutic adjunct to our current treatment approach to AML. Results from near future clinical trials will answer the question whether the dual inhibitor of FLT3 and JAK2 will be a real successful targeted agent.

As the next generation sequence technology advanced, more genetic and molecular changes in cancers including AML were discovered ${ }^{[6]}$. Multiple different genetic changes may cooperate in cancers. New mutations in signaling pathway or alternate pathway will emerge when treated by only one specific target agent ${ }^{[7]}$. Thus, from a clini- 
cal point of view there are at least two important aspects in this study. First, the resistant of target therapy could be possible reversed by simultaneously blocking two or more signaling pathways. It indicates a new strategy to screen for agents for an effective targeted cancer therapy. Second, since there are a handful of FLT3, JAK2 inhibitors or other kinase inhibitors have already been discovered and are currently being developed for clinical trials. It is rationale to designing trials in a more effec- tive manner by combining one targeted drugs along with other agents that target alternate mechanisms of disease pathogenesis.

1 Wang ZY, Chen Z. Acute promyelocytic leukemia: from highly fatal to highly curable. Blood 2008; 111: 2505-15.

2 Fathi A, Levis M. FLT3 inhibitors: a story of the old and the new. Curr Opin Hematol 2011; 18: 71-6.

3 Stein BL, Crispino JD, Moliterno AR. Janus kinase inhibitors: an update on the progress and promise of targeted therapy in the myeloproliferative neoplasms. Curr Opin Oncol 2011; 23: 609-16.
4 Hart S, Goh KC, Novotny-Diermayr V, Hu CY, Hentze H, Tan YC, et al. Pacritinib (SB1518), a JAK2/ FLT3 inhibitor for the treatment of acute myeloid leukemia. Blood Cancer J 2011; 1: e44.

5 Hart S, Goh KC, Novotny-Diermayr V, Tan YC, Madan B, Amalini C, et al. SB1518, a novel macrocyclic pyrimidine-based JAK2 inhibitor for the treatment of myeloid and lymphoid malignancies. Leukemia 2011; 25: 1751-9.

6 Mardis ER, Ding L, Dooling DJ, Larson DE, McLellan $\mathrm{MD}$, Chen K, et al. Recurring mutations found by sequencing an acute myeloid leukemia genome. $\mathrm{N}$ Engl J Med 2009; 361: 1058-66.

7 Wagle N, Emery C, Berger MF, Davis MJ, Sawyer A, Pochanard $\mathrm{P}$, et al. Dissecting therapeutic resistance to RAF inhibition in melanoma by tumor genomic profiling. J Clin Oncol 2011; 29: 3085-96. 